\title{
Association between hydration status, hydration knowledge and fluid consumption during training among soccer players
}

\author{
Jusoh N. ${ }^{A B C D E}$, Salim S. ${ }^{A B C D E}$ \\ Faculty of Sport Sciences and Coaching, Universiti Pendidikan Sultan Idris, Malaysia
}

Authors' Contribution: A - Study design; B - Data collection; C - Statistical analysis; D - Manuscript Preparation; E - Funds Collection.

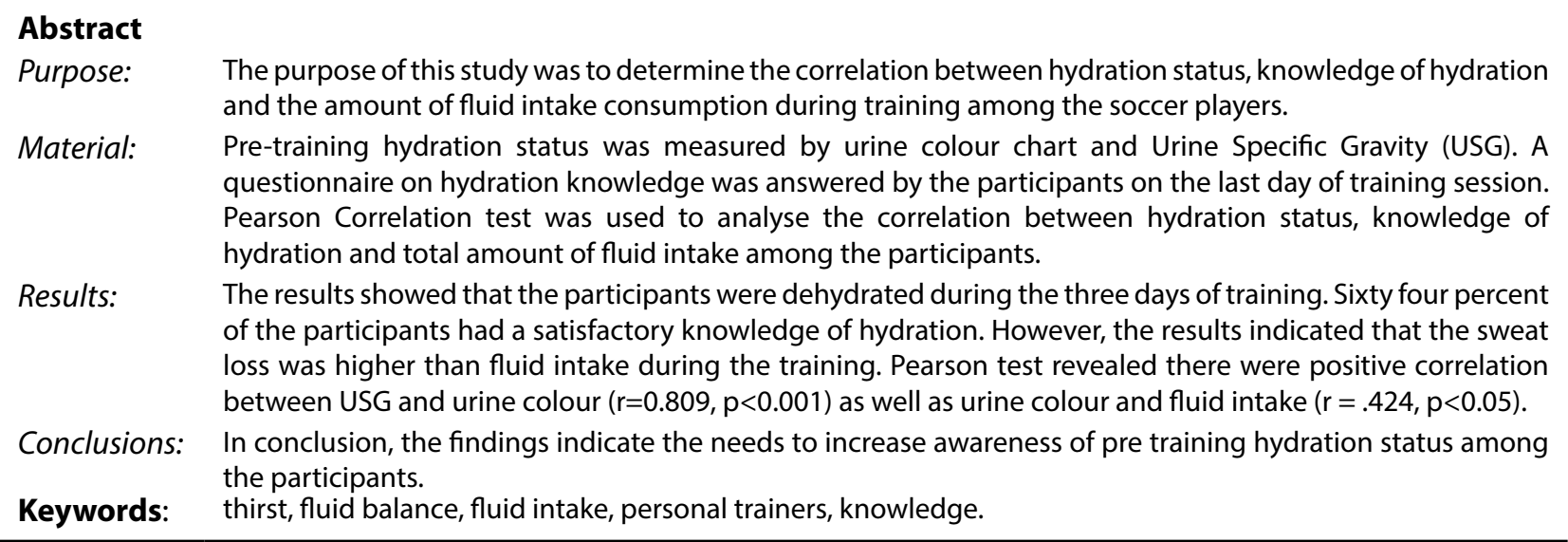

\section{Introduction}

Human body system needs fluid balance mechanism to ensure that all body system function well. When athletes train under the hot weather, the body's homeostatic balance can be disrupted [1]. Furthermore, during muscle contraction, the heat produced from the metabolism of the body which must be removed from the body to prevent hyperthermia. Besides, during training, body water could be lost through perspiration which leads to dehydration. According to Coyle [2], the effect of dehydration on the physiological functions and exercise performance is higher when the athletes were training under warm weather compared to cold weather.

If the level of dehydration increases, body temperature, heart rate and stroke volume will increase and jeopardize the sports performance. Therefore, it is very important for an athlete to ensure the fluid intake before, during and after exercise are sufficient. In fact, individuals who are active in sports require adequate energy and fluid intake to maintain normal hydration, electrolyte balance and variety of food choices that provide balanced nutrients for optimal performance [3]. Sawka et al. [4] stated that when dehydration occurs during training under warm weather, pressure on the physiology of the body increases and requires more effort to do the exercises. They also noted that the higher the level of dehydration, the performance of aerobic exercise will also be disrupted. Duffield, Mccall, Coutts and Peiffer [5] conducted the study on hydration, sweat and thermoregulatory responses among professional football players training in the heat found that the average rate of sweat loss during training under was between 0.7 - $1.5 \mathrm{Lh}^{-1}$ with weight loss between 1-5\%. This study supports the previous findings by Kilding, et

(c) Jusoh N., Salim S., 2019

doi:10.15561/20755279.2019.0104 al. [6] and Shirreffs et al. [7] in which they stated that the rate of sweat loss during exercise under hot weather was between 1.0-2.0 $\mathrm{Lh}^{-1}$ with weight loss between $1-3 \%$. Therefore, adequate fluid intake before and during training should be emphasized among athletes to maintain body fluid balance and sports performance. Furthermore, it is also to ensure that the athletes are not exposed to heat injury and health problems, especially heat illness. Apart from that, a study conducted by Ritz and Berrut [8] showed that minimal dehydration also disrupts some important aspects of cognitive function such as memory short ratio, sensitivity and concentration.

Despite the importance of proper hydration on sports performance, many athletes started their training $[9,10$, $11]$ or competition $[12,13]$ in hypohydrated state. This situation may be due to ignorance or lack of knowledge on the aspect of hydration. Normah [14] showed that the athletes in selected sport school in Perak had good score on hydration knowledge, yet they were dehydrated prior to the training session, suggesting they probably ignored the importance of proper hydration status for optimal training. Decher et al. [15] also showed that there was no correlation observed between hydration status and hydration knowledge among children who attended summer camp, and they speculated that other factors may contribute to the hypohydrated state among the children. Indeed, Nichols, Jonnalagadda, Rosenbloom, and Trinkaus [16] concluded that even though the athletes had knowledge on hydration, but they may not apply the knowledge to improve their hydration status. The application of hydration knowledge includes the sufficient fluid consumption before, during and after training as well as monitoring their hydration status using physiological and psychological signs.

Therefore, the purpose of this study was to examine 
the prevalence of dehydration among soccer players in Universiti Pendidikan Sultan Idris, Perak (UPSI) during three consecutive days of training. Besides, this study also sought to determine the relationship between hydration status, knowledge of hydration and fluid intake during training among these players. We hypothesized that there will be no significant correlation between hydration status and knowledge with fluid intake among the players. The study is crucial to increase knowledge and awareness of hydration status among UPSI soccer players, athletes, coaches, team managers and individuals who perform physical activity, especially in hot and dry environments such as in Malaysia.

\section{Materials and Methods}

\section{Participants}

A total of 25 male soccer players aged $22.3 \pm 1.1$ years, height $1.7 \pm 0.1 \mathrm{~m}$, body mass $63.3 \pm 8.9 \mathrm{~kg}$ and BMI $22.2 \pm 2.5 \mathrm{~kg} / \mathrm{m} 2$ volunteered to participate in this study. All participants were received verbal and written information about the study and consent form were obtained from them. The participants were also asked to complete the Health Screen Questionnaire prior to the trial. The participants were free from chronic diseases and did not have injuries within 6 months prior to the study. The study protocol was designed in accordance with the Helsinki Declaration and approved by the Faculty of Sports Science and Coaching Research Committee.

Procedure

The data collection were conducted during three consecutive days of training. Pre training and post training body weight was measured using digital weighing scale (Omron HN-283, Kyoto, Japan) accurate to $100 \mathrm{~g}$. Pre training and post training urine sample was also collected using a labelled urine collection container. The participants had also been instructed to collect any urine passed during training into the container given. Urine specific gravity (USG) and colour of urine were analysed using digital hand-held pocket refractometer (Atago PAL-10S, Japan), and urine colour chart [1] respectively. Digital hand-held pocket refractometer was calibrated after 10 times measurement and cleaned by using mineral water (Cactus, Selangor) and lens's tissue (Whatman, Maidstone, England). The cut-off point of USG $>1.020$ and urine colour numbers from 4 to 8 (Casa et al., 2000) were used to identify dehydration. The temperature and relative humidity during the three-days training was measured using Wet Bulb Globe Temperature (WBGT103, Japan).

During training, the participants were given only mineral water with the capacity of $1500 \mathrm{ml}$ (Cactus, Selangor) labelled with their name. They were told to drink ad libitum during the training period. The volume of consumed water was recorded before and after training using digital precision balance (Salter 1017, England) accurate to $1 \mathrm{~g}$. After training session on the third day of the study, the participants were asked to answer a set of modified Hydration Knowledge Questionnaire [16]. The result of Cronbach alpha showed the internal reliability value of 0.74 , suggesting that the modified questionnaire had high reliability. The questionnaire consists of two sections. Section A enquires about the participant demography and section B consists of 17 closed-ended questions about the hydration knowledge.

\section{Statistical analysis}

All data were analysed using Statistical Program for Social Sciences (SPSS) version 17.0 (Windows, SPSS. Inc., Chicago, IL). Kolmogorov-Smirnov test was utilized to check the normality of the data. Descriptive analysis was performed on the demographic data, hydration status (USG and urine colour), scores of hydration knowledge and volume of fluid intakes throughout 3 days of training. The findings were reported in mean, standard deviation $(\mathrm{SD})$, frequency $(\mathrm{N})$ and percentage (\%). Total score of hydration knowledge questionnaire had been categorized as Excellent (98\% - 100\%), Very Good (81.95\%-97.59\%), Good $(66.30 \%-81.94)$, weak $(66.29 \%-50.65 \%)$ and very weak (50.64\% and below).

Pearson Correlation Test was used to determine the relationship between hydration status, scores of hydration knowledge and volume of fluid consumption among the participants. The significant level was set at $\mathrm{P}<.05$.

\section{Results}

Table 1 shows the mean value of pre-training USG and urine colour of the participants during three days of training. The findings showed that the participants started their training in hypohydrated state on Day 1 and Day 3. The mean value of USG on Day 1 was $1.025 \pm 0.007$ and Day 3 was $1.022 \pm 0.009$. Likewise, urine colour on both Day 1 and day 3 reported the same value which was $4 \pm 1$.

Table 2 illustrates the level of hydration knowledge based on scores of 17 items. The results shows that $48 \%$ of the participants had good hydration knowledge and $16 \%$ scored satisfactorily. In contrast, only 36\% of the participants had poor to very poor knowledge in hydration. None of the participants scored very well for the questionnaire.

Data of fluid intake and sweat loss volume were used to calculate the fluid balance during the training. Table 3 shows the mean of the total fluid consumption, the amount of sweat loss and the difference between consumption of fluids and sweat loss during the 3-day training. The mean fluid intake were $1271.3 \pm 248.5 \mathrm{ml}, 1173.3 \pm 337.5$

Table 1. USG and urine colour during the three-day training (mean \pm SD)

\begin{tabular}{llll}
\hline Variable & Day $\mathbf{1}(\mathbf{n}=\mathbf{2 5})$ & Day 2(n=25) & Day 3(n=25) \\
\hline USG & $1.025 \pm 0.007$ & $1.019 \pm 0.010$ & $1.022 \pm 0.009$ \\
Urine Colour & $4 \pm 1$ & $3 \pm 1$ & $4 \pm 1$ \\
\hline
\end{tabular}


$\mathrm{ml}$ and $1241.3 \pm 232.3 \mathrm{ml}$ for Day 1, Day 2 and Day 3, respectively. Whereas, the mean sweat loss on the first, second and third days were $1759.3 \pm 418.5 \mathrm{ml}, 1573.3$ \pm 378.5 and $1877.3 \pm 450.6 \mathrm{ml}$. Thus, the difference between consumption of fluids and sweat loss during three days of training were $-488.0 \pm 364.4 \mathrm{ml},-400.0 \pm$ $285.8 \mathrm{ml}$ and $-636.0 \pm 396.2 \mathrm{ml}$. The results showed that the participants were in negative fluid balance because the drink consumption were less than the sweat loss during the three days of training.

Table 4 shows the correlation between hydration status, score of hydration knowledge and the amount of fluid consumption during training in UPSI Soccer Player. There was positive correlation observed between the USG and the urine colour $(\mathrm{r}=.809, p<0.001)$. Meanwhile, the there was correlation between USG and hydration knowledge score $(\mathrm{r}=-.099, p=.639)$ and the amount of fluid intake $(\mathrm{r}=.213, p=.308)$. In addition, urine colour and scores of hydration knowledge had weak correlation and $(\mathrm{r}=$ $.206, p=.324$ ). While the correlation between urine colour and the amount of fluid intake was significantly positive correlation $(\mathrm{r}=.424, p=.035)$. Hydration knowledge did not correlate well with the amount of fluid intake $(\mathrm{r}=.031$, $p=.883$ ).

\section{Discussion}

The results indicated that the participants were dehydrated before training, especially on the first and third day of training. They experienced varying level of dehydration ranges from minimal to serious dehydration. Our results was in agreement with many previous studies $[10,11,17]$ which found that the football players from different continents usually commenced their training in hypohydrated state.

Various factors may contribute to dehydration among the participants before training. The athletes may not consume suitable and adequate fluid intake during the early morning or several hours before training [18]. Sufficient amount of fluid intake may help the body to be in a normal state of hydration throughout the day. Accordingly, lack of knowledge on maintaining hydration status may also affect the hydration status of the players before training. Bland et al. [18] who studied the pretraining hydration status in athletes of National Collegiate Athletic Association (NCAA) suggested that the athletes should be given proper education on hydration strategy and should be encouraged to practice hydration schedule to ensure optimal hydration and well beings. In addition, the intensity and frequency of training exercises on a daily basis is also one of the factors that may cause the dehydration among the participants. Excessive training load may dehydrate the body, even after training. If the hydration status does not return to normal state, dehydration will be experienced by the athletes before training the following day. Furthermore, dietary intake and medications may also affect the day-to-day hydration

Table 2. Level of hydration knowledge of the participants (frequency and percentage)

\begin{tabular}{lll}
\hline Hydration knowledge & Frequency $\mathbf{( n = 2 5 )}$ & Percentage $(\mathbf{n}=\mathbf{2 5})$ \\
\hline Very Good & 0 & 0 \\
Good & 12 & 48 \\
Satisfactory & 4 & 16 \\
Poor & 7 & 28 \\
Very Poor & 2 & 8 \\
\hline
\end{tabular}

Table 3. Fluid balance during three consecutive days of training (mean \pm SD)

\begin{tabular}{llll}
\hline Variables & Day $\mathbf{1}(\mathbf{n}=\mathbf{2 5})$ & Day $\mathbf{2}(\mathbf{n}=\mathbf{2 5})$ & Day $\mathbf{3}(\mathbf{n}=\mathbf{2 5})$ \\
\hline Total of fluid consumption $(\mathrm{ml})$ & $1271.3 \pm 248.5$ & $1173.3 \pm 337.5$ & $1241.3 \pm 232.3$ \\
Amount of sweat loss $(\mathrm{ml})$ & $1759.3 \pm 418.5$ & $1573.3 \pm 378.5$ & $1877.3 \pm 450.6$ \\
Difference between fluid consumption & $-488.0 \pm 364.4$ & $-400.0 \pm 285.8$ & $-636.0 \pm 396.2$ \\
and sweat loss $(\mathrm{ml})$ & & & \\
\hline
\end{tabular}

Table 4. Correlation between hydration status, knowledge of hydration and fluid consumption during training in UPSI soccer players $(n=25)$

\begin{tabular}{lllll}
\hline Variables & USG & Urine colour & $\begin{array}{l}\text { Hydration } \\
\text { Knowledge }\end{array}$ & Fluid consumption \\
\hline USG & - & & \\
Urine colour & $r=0.809^{* *}$ & - & - \\
Hydration knowledge & $r=-.099$ & $r=-.206$ & $r=.031$ & - \\
Fluid consumption & $r=.213$ & $r=.424^{*}$ & $r$ \\
\hline
\end{tabular}

** Correlation is significant at $P<0.01 ; \quad$ *Correlation is significant at $P<0.05$ 
status of the athletes $[19,20,21]$. Therefore, early detection of hydration status is very important to maintain performance during training. According to Maughan and Shirreffs [19], in order to avoid deficiency of fluid and salt in the body, the athletes are encouraged to keep themselves euhydrated before training. This is because when hypohydration occurs, the physiological changes will occur and may expose the athletes to heat illnesses including heat stroke [4, 22, 23, 24].

The second major findings of the present study was that hydration knowledge among the participants are yet to reach excellent level or completely acquired very well on their body hydration. Table 2 illustrates that more than $50 \%$ of the participants had a good and satisfactory knowledge in hydration, but most of the participants experienced dehydration particularly on the third day of the training. Supposedly, if the participants have sufficient knowledge about hydration, they should be alert on their hydration status, especially before training. This statement was further supported by Nichols et al., [16], who suggested that lack of knowledge among athletes makes them unaware in maintaining their normal hydration status. But this view does not apply to the participants in our study. Thus, it indicates that despite having a good knowledge in hydration, but they might not apply the knowledge to keep them hydrated especially before commencing the training session. Our result was similar to the study conducted by Decher and colleagues [15] on hydration status, knowledge, and behaviour in youths at summer camp in the US. They reported that the participants of the camp could identify their hydration status, but they failed to translate hydration knowledge to an effective hydration strategy. This was proved by the results of USG and urine osmolality (Uosm) of the participants, which ranges from minimal to significant dehydration during the four-day camp.

Furthermore, based on the feedback of the individual item in the questionnaire, most of the participants answered correctly on questions of general hydration. For example, the following item categorized as general hydration knowledge "It is important for individuals who are active in sports drinking water during training and competition" was answered correctly by all participants. However, many of them did not answer correctly on the questions related to the guidelines of fluid intake of National Athletic Trainers Association (NATA) and the American College of Sports Medicine (ACSM) position stands before, during and after training or competition. Position stands from both ACSM [4] and NATA [23] provide guidelines on fluid and electrolyte needs and replacement as well as the impact of the imbalance on exercise performance. To illustrate, $52 \%$ of the participants gave the wrong answer regarding the statement "individuals who are active in sports should drink sports drinks in two hours after training" which was recommended by ACSM and NATA to ensure optimal exercise performance. The results of our study was in agreement with other studies $[25,26,27]$. These study revealed the athletes have little understanding of the positive effects of sports drinks to maintain proper hydration and exercise performance especially in intermittent and endurance events. Therefore, they suggested that the athletes should be educated regarding the beneficial role of carbohydrate and electrolytes in sports drinks to help regain energy and maintain normal hydration status. Accordingly, the researchers recommended that knowledge about consuming proper sports drinks should also be given to the coaches and athletic trainers because they are partly responsible for the athletes' performance.

To conclude, a total of $64 \%$ of the participants in our study possess a good hydration knowledge, especially in terms of replacement of body fluids. However, they are confused about a specific drink to be taken before, during and after training or competition. They also perplexed in identifying the correct signs for dehydration. Therefore, it is crucial for the coaches and team managers to provide correct and relevant information regarding hydration to the athletes to ensure proper hydration status during training and competition. The findings of the present study showed that the participants consumed water to replace fluids loss during training. However, the amount of fluid intake were less than the fluid loss through sweat during three consecutive days of training (Table 3). Our results support the previous studies of the fluid balance among soccer players during training and competition $[13,17$, $28,29,30]$. These studies concluded that the athletes rarely consume beverage on par with the amount of sweat loss even though the beverages were readily available, leading to hypohydration during training and competition.

The findings from our study suggests that the participants need not merely to drink fluid during training and competition, but more importantly, they should consume adequate amount of fluids to replace sweat loss to maintain proper hydration. Furthermore, [16] recommended that the coaches and team managers should increase the availability of drinking stations, provide bottled drinks and monitor the weight changes before and after training in order to promote sufficient fluid consumption among athletes. Total fluid requirement varies among athletes. Fluid replacement protocols recommended by ACSM and NATA can only be used as a general guideline for the athletes to consume fluids correctly. The actual amount of drinks need to be replaced during training and competition depends on the individual sweat loss. The athletes who consume fluids which is not based on individuality are prone to incidence of dehydration because the amount of sweat loss during training is different for each athlete [29].

Results of Pearson product-moment test showed that only USG and urine colour $(\mathrm{r}=.809, p<0.001)$ and urine colour and the amount of fluids intake ( $\mathrm{r}=.424, p<.035)$ had significantly positive correlations. A strong correlation observed between USG and urine colour to indicate hydration status in our study was similar to the study done by [31]. The results of the study showed that there was a strong relationship between urine osmolality $(\mathrm{r}=.80)$ and USG $(\mathrm{r}=.82)$ with urine colour. Therefore, the use of USG and urine colour in the present study is adequate 
to estimate hydration status of the participants prior to training. Indeed, USG is more accurate and practical than other expensive methods to measure hydration status $[32,33]$. Furthermore, through the observation of urine colour, the athletes can easily determine when they should hydrate themselves during exercise. Even though the urine colour does not provide the same accuracy and precision compared to USG and urine osmolality, but urine colour is practical for field research setting and athletes who do not require high accuracy for estimating hydration status [20].

Another significant correlation was detected between urine colour and fluid consumption, but the value did not reach strong correlation. The result violates our speculation whereby when the fluid intake increases, the urine colour supposedly become lighter which indicates euhydration. However, in our study fluid consumption was not sufficient to replace sweat loss as showed in Table 5, which might explain the high incidence of pre training dehydration for three consecutive days despite voluntarily taking fluid during training sessions. When the participants trained in a state of dehydration, it is difficult for their body to maintain normal hydration level because of their resting time and the provision of adequate water during training in the field. This statement was in agreement with [34] whereby they found that if the athletes are dehydrated prior to training, they experienced various difficulties to hydrate themselves during training.

Our study also indicated that there were no strong correlation between both hydration indices (USG and urine colour) with hydration knowledge. Most of the participants were experienced high incidence of dehydration ranges from minimal to serious level despite scored well in hydration knowledge test. The correlation result implies that having knowledge does not necessarily translate into good hydration strategy among the participants. Our results support the findings [35] which revealed that no relationship exists between knowledge and practices on hydration and fluid replacement among endurance athletes in National University of Malaysia. However, a study by [16] on knowledge, attitude and behaviour regarding hydration in collegiate athletes in the US showed otherwise. The study found a significant and positive correlation between the scores of knowledge, attitude and behaviour among the participants. Although the correlation was low, it shows that when hydration knowledge and attitudes score increased, score on behaviours also increased. Thus it is important for the coaches and managerial team not only to educate the athletes on hydration, but also highlight the need to create a positive attitude and behaviour so that the athletes can apply the knowledge to strategize the fluid replacement for optimal sports performance.

\section{Conclusion}

The obvious findings emerged from this study is that a large numbers of UPSI soccer players are dehydrated before training. The athletes should monitor and be responsible for handling and preventing dehydration before training. The athletes should not depend on their coach or team manager to remind about their hydration practices. Experts have provided recommendations regarding the importance for the athletes to identify rehydration strategies on their own [19]. The recommendation includes encouraging the players to measure their body weight before and after training sessions in different climatic conditions, as well as intensity and duration of exercise to estimate their sweat loss. Weight loss exceeds $1-2 \%$ of body weight indicates that the athletes do not consume enough fluids and they should drink accordingly. Therefore, the athletes should know the right amount of fluid that is adequate to replace excessive sweat loss during training to prevent dehydration on the subsequent days of training.

The athletes also need to improve on the knowledge about dehydration so that they can be more responsive to the sign of dehydration before training. The players will have a risk of dehydration if their urine production is less than daily observation. This means that, if urine production is less and the colour becomes darker, the athletes should increase the fluid intake until their urine production increases and the urine colour changes to pale yellow. The athletes should be encouraged to think and observe why and when they should consume more fluid attributed to the loss of sweat and urine production. Coaches and managerial team are the backbone of the success of a team or an athlete. However, the strategy to get the best performance for training or competition will not complete if they ignored the nutrition especially on hydration strategy. Therefore, it is suggested that the coaches or team manager provide a record of the data collection and constructive suggestions on the improvement of hydration practices for the players.

In conclusion, the athletes, coaches and managerial team should do a routine monitoring of hydration status before the athletes start their training. Besides, having knowledge without proper hydration practices may not prevent the athletes from risks of dehydration and performance deterioration. Therefore, investigations into the interventions to improve fluid replacement practices among exercising individuals is warranted.

\section{Acknowledgements}

The authors gratefully acknowledge Mr. Khairuddin Bin Abdul Majid, a coach for UPSI soccer team who had given permission and support to carry out this study. A special thanks is also extended to all 25 UPSI soccer players for their support and cooperation to voluntarily participate in this study.

\section{Conflict of interest}

The authors declare no conflict of interest. 


\section{References}

1. Armstrong LE. Performing in extreme environments. Champaign, IL: Human Kinetics; 2000.

2. Coyle EF. Fluid and fuel intake during exercise. Journal of Sports Science, 2004;22(1): 39-55. https://doi.org/10.1080/0264041031000140545

3. Laquale K. Nutritional needs of the recreational athlete. Athletic Therapy Today, 2009;14(1), 12-15. https://doi.org/10.1123/att.14.1.12

4. Sawka MN, Burke LM, Eichner ER, Maughan RJ, Montain SJ, Stachenfeld NS. American College of Sports Medicine Position Stand. Exercise and fluid replacement. Medicine and Science in Sports and Exercise, 2007;39(2), 377-390. https://doi.org/10.1249/mss.0b013e31802ca597

5. DuffieldR,MccallA,CouttsAJ,PeifferJJ.Hydration, sweatand thermoregulatory responses to professional football training in the heat. Journal of Sports Science, 2012;30(10), 957-965. https://doi.org/10.1080/02640414.2012.689432

6. Kilding AE, Tunstall H, Wraith E, Good M, Gammon C, Smith C. Sweat rate and sweat electrolyte composition in international female soccer players during game specific training. International Journal of Sports Medicine, 2009;30(6), 443-447. https://doi.org/10.1055/s-0028-1105945

7. Shirreffs SM, Aragon-Vargas LF, Chamorro M, Maughan RJ, Serratosa L, Zachwieja JJ. The sweating response of elite professional soccer players to training in the heat. International Journal of Sports Medicine, 2005;26 (2), 90-95. https://doi.org/10.1055/s-2004-821112

8. Ritz P, Berrut G. The importance of good hydration for day-today health. Nutrition Reviews, 2005;63(6), S6- S13. https://doi.org/10.1301/nr.2005.jun.S6-S13

9. Magal M, Cain RJ, Long JC, Thomas KS. Pre-practice hydration status and the effects of hydration regimen on collegiate division III male athletes. Journal of Sports Science and Medicine, 2015;14, 23-28.

10.Castro-Sepulveda M, Astudillo J, Letelier P, ZbindenFoncea H. Prevalence of dehydration before training sessions, friendly and official matches in elite female soccer players. Journal of Human Kinetics, 2016;50, 79- 84. https://doi.org/10.1515/hukin-2015-0145

11.Phillips SM, Skyes D, Gibson N. Hydration status and fluid balance of elite European youth soccer players during consecutive training sessions. Journal of Sports Science and Medicine, 2014; 13, 817-822.

12. Chapelle L, Tassignon B, Aerenhouts D, Mullie P, Clarys P. The hydration status of elite youth female soccer players during an official tournament. Journal of Sports Medicine and Physical Fitness, 2017; 57(9), 1186-1194. https://doi.org/10.23736/S0022-4707.16.06527-0

13.Da Silva RP, Mundel T, Natali A, Bara Filho MG, Alfenas RC, Marins JC et al. Pre-game hydration status, sweat loss, and fluid intake in elite Brazilian young male soccer players during competition. Journal of Sports Science, 2012; 30(1), 37-42. https://doi.org/10.1080/02640414.2011.623711

14. Normah J. Relationship between hydration status, hydration knowledge and fluid intake behaviour among school athletes of selected Perak sport schools. Journal of Sports Science and Physical Education, 2014;3(1), 11-19.

15.Decher NR, Casa DJ, Yeargin SW, Ganio MS, Levreault ML, Dann CL, et al. Hydration status, knowledge, and behavior in youths at summer sports camps. International Journal of Sports Physiology Performance, 2008; 3(3), 262-278. https://doi.org/10.1123/ijspp.3.3.262

16.Nichols PE, Jonnalagadda SS, Rosenbloom CA, Trinkaus M.
Knowledge, attitude and behaviours regarding hydration and fluid replacement of collegiate athletes. International Journal of Sport Nutrition and Exercise Metabolism, 2005;15,515-527. https://doi.org/10.1123/ijsnem.15.5.515

17.Kurdak SS, Shirreffs SM, Maughan RJ, Ozgunen KT, Zeren C, Korkmaz S, et al. Hydration and sweating responses to hot-weather football competition. Scandinavian Journal of Medicine and Science in Sports, 2010;20 (suppl. 3), 133-139. https://doi.org/10.1111/j.1600-0838.2010.01218.x

18. Bland EG, Poule KA, Volpe SL. Estimation of Pre practice Hydration Status of National Collegiate Athletic Association Division I Athletes. Journal of Athletic Training, 2009; 44(6), 624-629. https://doi.org/10.4085/1062-6050-44.6.624

19.Maughan RJ, Shirreffs SM. Development of individual hydration strategies for athletes. International Journal of Sport Nutrition and Exercise Metabolism, 2008;18(5), 457-472. https://doi.org/10.1123/ijsnem.18.5.457

20.Armstrong LE. Hydration assessment techniques. Nutrition Review, 2005; 63(6), S40-S54. https://doi.org/10.1301/nr.2005.jun.S40-S54

21.Thompson WG. Things that go red in the urine; and others that don't. The Lancet, 1996; 347(8993), 5-6. https://doi.org/10.1016/S0140-6736(96)91551-2

22.Armstrong LE, Casa DJ, Millard-Stafford M, Moran DS, Pyne SW, Roberts WO. American College of Sports Medicine Position Stand: Exertional heat illness during training and competition. Medicine and Science in Sports and Exercise, 2007;39(3), 556-572. https://doi.org/10.1249/MSS.0b013e31802fa199

23.Casa DJ, Armstrong LE, Hillman SK, Montain SJ, Reiff RV, Rich BE, et al. National athletic trainers' association position statement: Fluid replacement for athletes. Journal of Athletic Training, 2000; 35(2), 212-224.

24.Armstrong LE, Soto JAH, Hacker FT, Casa DJ, Kavouras SA, Maresh CM. Urinary indices during dehydration, exercise and rehydration. International Journal Sport Nutrition, 1998; 8, 345-355. https://doi.org/10.1123/ijsn.8.4.345

25.Felder JM, Burke LM, Lowdon BJ, Cameron-Smith D, Collier, GR. Nutritional practices of elite female surfers during training and competition. International Journal of Sport Nutrition, 1998;8(1), 36-48. https://doi.org/10.1123/ijsn.8.1.36

26.Jonnalagada SS, Rosenbloom CA, Skinner R. Dietary practices, attitudes, and physiological status of collegiate freshman football players. Journal of Strength Conditioning Research, 2001;15(4), 507-520.

27.Jonnalagada SS, Rosenbloom CA, Skinner R. Nutrition knowledge of Collegiate athletes in Division I National Collegiate Athletic Association institution. Journal of American Dietetic Association, 2002; 102(3), 418-420. https://doi.org/10.1016/S0002-8223(02)90098-2

28.Gordon RE, Kassier SM, Biggs C. Hydration status and fluid intake of urban, underprivileged South African male adolescent soccer players during training. Journal of International Society of Sports Nutrition, 2015; 12:21. https://doi.org/10.1186/s 12970-015-0080-0

29.Maughan RJ, Merson SJ, Broad NP, Shireff SM. Fluid and electrolyte intake and loss in elite soccer players during training. International Journal of Sport Nutrition and Exercise Metabolism, 2004; 14(3), 333-379. https://doi.org/10.1123/ijsnem.14.3.333

30.Burke LM, and Hawley JA. Fluid balance in team sports: Guidelines for optimal 
practices. Sports Medicine, 1997;24(1), 38-54. https://doi.org/10.2165/00007256-199724010-00004

31.Armstrong LE, Maresh CM, Castellani JW, Bergeron MF, Kenefick RW, LaGasse KE, et al. Urinary indices of hydration status. International Journal of Sport Nutrition, 1994;4(3), 265-279. https://doi.org/10.1123/ijsn.4.3.265

32.Eberman LM, Cleary MA. Comparison of refractometry, urine color, and urine reagent strips to urine osmolality for measurement of urinary concentration. Athletic Training in Sports Health Care, 2009; 1(6), 267-271. https://doi.org/10.3928/19425864-20091020-01
33.Oppliger R, Bartok C. Hydration testing of athletes. Sports Medicine, 2002;32(15), 959-971. https://doi.org/10.2165/00007256-200232150-00001

34. Williams CA, Blackwell J. Hydration status, fluid intake, and electrolytelossesinyouth soccerplayers. InternationalJournal of Sports Physiology and Performance, 2012; 7(4), 367-374. https://doi.org/10.1123/ijspp.7.4.367

35.Razalee S, Mohamad Mustafa M, Zalifah MK. Knowledge, attitude and fluid replacement among endurance sports athletes in national University of Malaysia (UKM). Pakistan Journal of Nutrition, 2015;14(10), 658- 665. https://doi.org/10.3923/pjn.2015.658.665

\section{Information about the authors:}

Jusoh N. (Corresponding author); http://orcid.org/0000-0003-4420-1763; normah@fsskj.upsi.edu.my; Universiti Pendidikan Sultan Idris; Tanjung Malim, 35900 Perak, Malaysia.

Salim S.; http://orcid.org/0000-0003-4050-9461; shaniza@mrsmtaiping.edu.my; Universiti Pendidikan Sultan Idris; Tanjung Malim, 35900 Perak, Malaysia.

\section{Cite this article as:}

Jusoh N, Salim S. Association between hydration status, hydration knowledge and fluid consumption during training among soccer players. Physical education of students, 2019;23(1):23-29.

https://doi.org/10.15561/20755279.2019.0104

The electronic version of this article is the complete one and can be found online at: https://sportedu.org.ua/index.php/PES/issue/archive

This is an Open Access article distributed under the terms of the Creative Commons Attribution License, which permits unrestricted use, distribution, and reproduction in any medium, provided the original work is properly cited http://creativecommons.org/licenses/by/4.0/deed.en

Received: 07.02.2019

Accepted: 21.02.2019; Published: 28.02.2019 